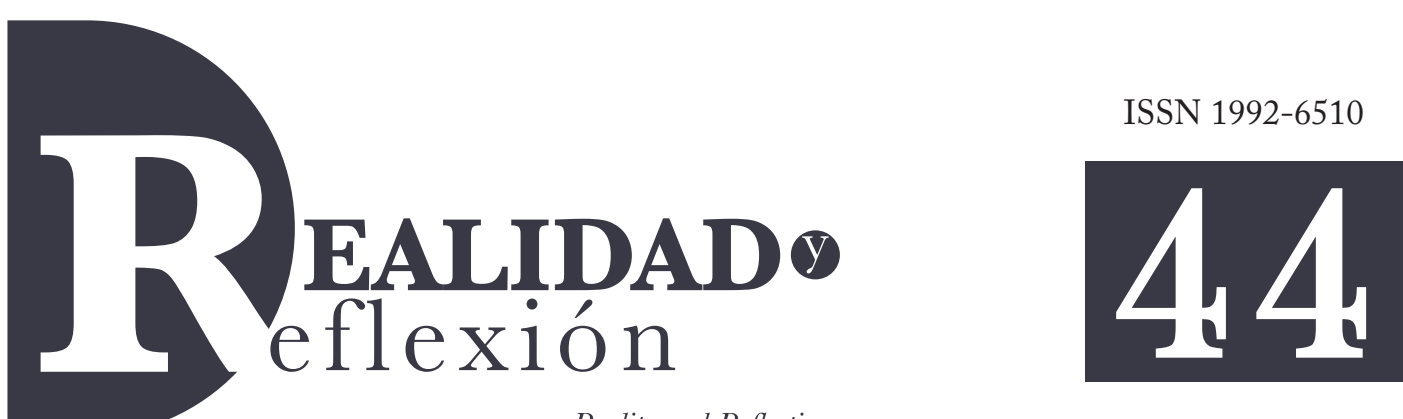

Reality and Reflection

Año 16, Nº 44, San Salvador, El Salvador, Centroamérica. Revista Semestral Julio-Diciembre 2016

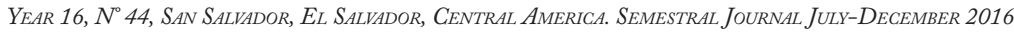

\title{
Planeación prospectiva: Escenarios y clústeres normativos
}

\section{Prospective Planning: scenario classifications and prospective clusters}

\author{
Francisco Álvarez Echeverría \\ Ingeniero Constructor Militar, \\ Máster en Ingeniería y Doctor en Ingeniería de Sistemas \\ franlve@yahoo.com.mx
}

\section{RESUMEN}

El presente artículo presenta algunos principios de construcción de escenarios prospectivos, proponiéndose a su vez una clasificación de éstos, de acuerdo a su forma de construcción, al tipo de planeación a utilizarse y de acuerdo a su intención de desarrollo. Así mismo, se plantea la construcción de clústeres de variables para la construcción de escenarios prospectivos a través de la utilización de lazos causales con el objeto de estructurar el entramado base para la proyección de tendencias. De igual manera, se mencionan los enfoques más importantes, utilizados para la construcción de escenarios prospectivos.

Palabras clave: planeación prospectiva, clústers, lazos causales.

\section{ABSTRACT}

This paper presents some principles of construction of prospective scenarios, proposing to turn their classification according to the form of construction, the type of planning used and according to their intention to development. Likewise, cluster construction variables for building future scenarios arise through the use of causal loops in order to structure the network basis for the projection of trends. In the same way the paper makes mention of the most important approaches used for the construction of prospective scenarios.

Keywords: scenario planning, clusters, causal loops. 


\section{Introducción}

Los estudios del futuro datan de varios siglos atrás, no sólo en forma de ficción sino también a través de técnicas y metodologías que permitían extrapolar resultados de un presente hacia un futuro o estadio posterior (Polak, 1973 y Miklos, 2007). No es sino hasta principios del siglo veinte cuando se presenta el primer intento formal, en donde William Ogburn (1937) a través de su reporte de Tendencias tecnológicas y política nacional, inclusión de las implicaciones sociales de los nuevos eventos enfatiza la importancia de los esfuerzos nacionales para llevar a cabo un ajuste en los planes, de conformidad con las situaciones cambiantes del entorno (Miklos, 2007).

Sin embargo, es hasta en las postrimerías de la Segunda Guerra Mundial donde son utilizados los escenarios de forma intensiva, y tornándose en ocasiones en meras probabilidades de ocurrencia de eventos específicos -es entonces cuando los escenarios empiezan a convertirse en un campo especializado debido a la guerra misma- (Miklos et al, 2007). Finalizada la Segunda Guerra Mundial surgieron diversas instituciones y corporaciones (así como estudios especializados en el tema) como RAND Corporation, Centre d'etudes Prospectives, Futuribles, Hudson Institute, entre otros (The Centre of Strategic Business Studies, 1999). Dichas instituciones y/o corporaciones fueron fundadas por actores, que a la fecha han contribuido de manera fundamental en la construcción teórica y práctica para el desarrollo de escenarios, entre estos autores se tienen a Herman Kahn, Gaston Berger, Andre Clement Decoufie, Pierre Masse, Bertrand de Louvenel, Olaf Helmer, Norman Dalkey, Pierre Wack, Robert Dubin, Hasan Ozbekhan Anthony
Wiener, los cuales han permitido el desarrollo de un nuevo tipo de pensamiento con características holísticas y sistémicas (Miklos, 2007).

\subsection{El proceso de planeación y los escenarios}

Dentro de la etapa del análisis en la toma de decisiones, las organizaciones requieren tomar decisiones que les permita afrontar los posibles cambios dentro del entorno competitivo (Van Der Heijden, 2006). El propósito de la estrategia es desarrollar una serie de políticas que conlleven a los individuos involucrados (directivos, personal, etc.) a direccionar el comportamiento individual como colectivo, con el objeto de que todo el sistema funcione de la mejor manera posible ante las diversos eventos que pudieran acontecer. El problema que se presenta a partir de lo anterior es el cómo desarrollar y alcanzar dicha estrategia cuando existen de por medio la incertidumbre y la ambigüedad dentro de un contexto dado. Igualmente, dentro del futuro (próximo o lejano) es posible afirmar que no todas las cosas/hechos/ situaciones son totalmente impredecibles.

Es un hecho que la idea del proceso de planeación del futuro se encuentra fundamentalmente basada sobre la impredecibilidad del futuro; sin embargo existe forma de identificar e inferir algunos aspectos de la realidad futura, a través de la observación y la asociación de eventos aparentemente inconexos. No obstante, al mismo tiempo, lo anterior conlleva a la obtención de un aprendizaje continuo y a la creación sistema de superficie-respuesta dinámica-continua ante las situaciones que se presenten posteriormente; en donde el comportamiento, las decisiones y la ejecución de la estrategia, por parte del ente en cuestión (individual, colectivo y/o 
organizacional) juegan un papel determinante en el éxito de la implementación estratégica.

Desde este contexto los escenarios normativos han dejado de ser una técnica, para convertirse en un método (Van Der Heijden, 2006). Inicialmente, el análisis de los escenarios era una extensión de la predicción y control dentro de la planeación, en la cual la línea de tiempo involucrada era sustituida por un modelo probabilístico, con el objeto de medir numéricamente la plausibilidad de los diferentes escenarios. Hoy en día los escenarios son mucho más que una mera proyección probabilística; más bien son una metodología de planeación en donde se ven involucradas la planeación estratégica, la planeación contingente y la planeación operativa; transformándose así en un análisis sistémico y holístico dentro del proceso de planeación y la toma de decisiones. Es por ello que la planeación a través de escenarios incluye no sólo la administración de los recursos, sino también estos componentes mismos de las estrategias posibles:

- El valor social/consumidor creado por la organización/entidad.

- Los recursos distintivos y competencias hacia el interior de la organización (creación del valor de la corporación).

- Los lazos de reforzamiento dentro de la retroalimentación, los cuales a su vez se tornan en ideas dentro de la sostenibilidad de las capacidades productivas y de sobrevivencia de la organización.

El entorno de la organización, entonces, afecta en diversos grados la actuación de ésta en un contexto particular, ya que éste (el entorno) se compone de factores exógenos, y la empresa/ entidad/organización en la mayoría de las ocasiones, carece de poca o de ninguna influencia sobre éstos. Al no tenerse control sobre dichos factores externos, la capacidad de respuesta ante los cambios que se presentan en el entorno resulta fundamental para la sobrevivencia de la empresa al tratar de anticiparse al futuro.

\subsection{Enfoques para la construcción de escenarios}

Los escenarios pueden desarrollarse principalmente en al menos tres campos. El primero, cuando lo que se requiere es la consolidación de las finanzas corporativas, en lo referente a la adquisición de nuevas formas o métodos de hacer las cosas. El segundo, la elección del tipo de cambio a incorporar dentro del proceso de creación de un negocio (productos primarios, producción, distribución).Y el tercero, cuando se requiere conocer, de alguna forma o de otra, las necesidades y los productos futuros de los consumidores dentro de un ambiente dinámico plagado de riesgos e incertidumbres. Y es en este punto donde se puede visualizar que existen diversos tipos de escenarios desarrollados bajo diversos enfoques, como puede observarse en el trabajo de Vergara et al. (2010), en donde se pueden identificar fácilmente alrededor de 18 modelos/metodologías para la construcción de escenarios, dependiendo del objeto focal y la intención de su utilización.

Con base en lo anterior existen al menos tres enfoques que sirven de referentes en la construcción de escenarios futuros: (1) El Enfoque francés; (2) El Enfoque norteamericano; y el (3) El Enfoque nórdico. 
Cabe señalar que, dentro de estos enfoques, gira a su alrededor una serie de métodos $\mathrm{y}$ técnicas de apoyo para la construcción de escenarios, así como un número importante de artículos, trabajos y proyectos realizados; dando lugar a una teoría-práctica sólida y desarrollada, originando que dichos enfoques dejen de ser una mera propuesta metodológica, convirtiéndose así en una herramienta calibrada a través del tiempo.

\subsubsection{El Enfoque francés}

Dentro del Enfoque francés el método de los escenarios se concibe como una vía para construir representaciones de los futuros posibles, así como el camino que conduce a la consecución de sus objetivos y metas planeados con anterioridad. El objetivo de estas representaciones es identificar las tendencias dominantes y los gérmenes de ruptura del entorno general y competencial de una organización (Godet et al, 2000). (Ver Figura n. ${ }^{\circ}$ 1).

*MICMAC: Matrices de Impactos Cruzados Aplicada a la Clasificación de variables; MACTOR: es un método de análisis de juego de actores; y MORPHOL SMIC: método que tiende a explorar de manera sistemática los futuros posibles a partir del estudio de todas las combinaciones resultantes de la descomposición de un sistema.

Para la construcción de escenarios, en primera instancia, se construye un conjunto de representaciones del estado actual del sistema constituido por la empresa/organización/ente y su entorno; para ello se requiere elaborar un estudio retrospectivo profundo y tan detallado como sea posible, siendo importante (Godet et al, 2000): (1) Delimitar el sistema y su entorno; (2) Determinar las variables esenciales; y (3) Analizar la estrategia de actores.

Posteriormente, se empiezan a preparar los futuros posibles a través de una lista de hipótesis que deberán reflejar una tendencia, el mantenimiento o decaimiento de ésta, el surgimiento de una nueva tendencia y/o su ruptura. Una vez preparados los insumos se procede a desarrollar la evolución del sistema -fase diacrónica (Godet et al, 2000)-. Aunque Godet et al (2000 y 2006) presentan un método de escenarios lógico y coherente, no es necesario recorrerlo de principio a fin o desarrollarlo de manera secuencial (ver Figura 1); todo depende del grado de conocimiento del sistema en estudio de los objetivos e intereses que se persigan al momento de realizar el estudio y el dominio del método prospectivo.

\subsubsection{E1 Enfoque norteamericano}

Si bien es cierto que la metodología general de la escuela norteamericana podría estar asociada a Peter Swhartz (1996) en primera instancia, aunque existen otros autores que de una manera $\mathrm{u}$ otra han contribuido con el desarrollo metodológico, ya sea con otras variantes o a través de la incorporación de herramientas como Van der Heijden (2006), Ringland (2002), Fahey y Randall (1998, entre otros. Dentro de estos aportes o contribuciones importantes se encuentran los de Kees Van der Heijden (2006), entre los cuales se tienen (CSBS, 1999) el desarrollo de la idea del negocio y las conversaciones estratégicas. (Ver Figura n. ${ }^{\circ}$ 2). 


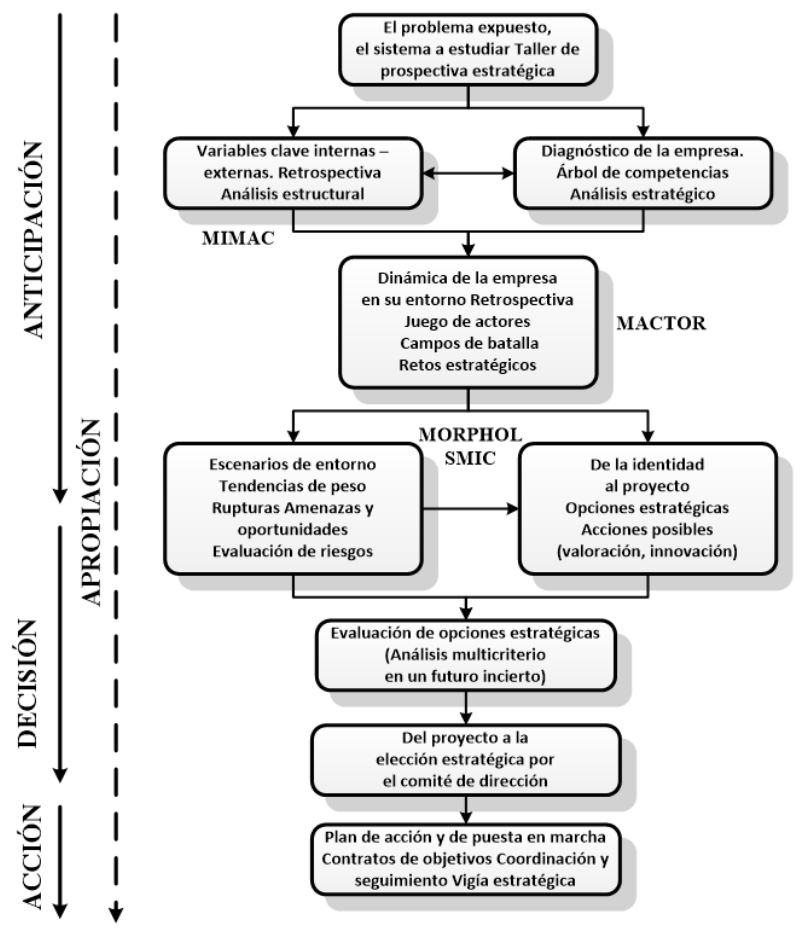

Figura n..$^{\circ}$. Enfoque francés para la construcción de escenarios. Fuente: Adaptado de Godet et al, 2006 y Godet 2000.

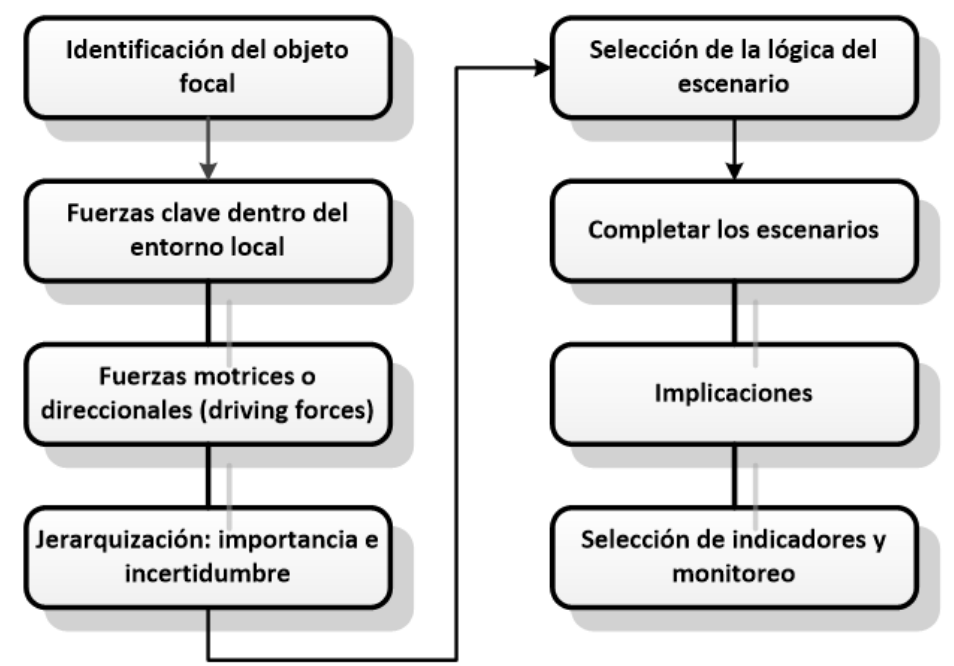

Figura n. $^{\circ}$ 2. Enfoque norteamericano para la construcción de escenarios. Fuente: Elaboración propia 
En este tipo de construcción (ver Figura 2) se tiene una serie de pasos que se deben considerar para el desarrollo e implementación de estos escenarios prospectivos (Swhartz, 1996): (1) Identificación del objeto focal o el objeto de la decisión; (2) Fuerzas clave dentro del entorno local; (3) Fuerzas motrices o direccionales (driving forces); (4) Jerarquización, importancia e incertidumbre; (5) Selección de la lógica del escenario; (6) Completar los escenarios; (7) Implicaciones; y finalmente, (8) Selección de indicadores y monitoreo.

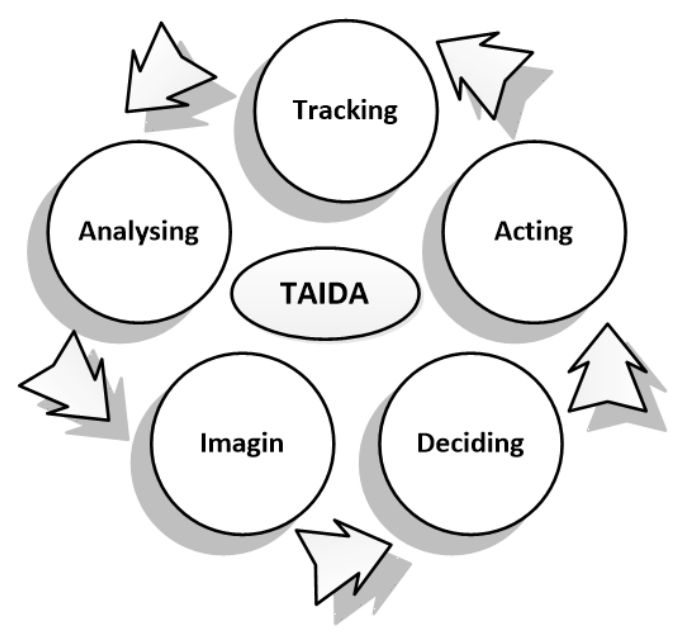

Figura n. $^{\circ}$ 3. El Enfoque nórdico para la construcción de escenarios.

Fuente: Elaboración propia.

\subsubsection{El enfoque nórdico}

En la construcción de escenarios existen diversas técnicas y métodos que han mostrado su eficacia en la creación de un marco de referencia para su elaboración. En años recientes se ha desarrollado una metodología de planeación de escenarios, como consecuencia de la conformación de una nueva escuela prospectiva liderada por Mats Lindgren y Hans Bandhold (2009). En este enfoque se utiliza una técnica llamada TAIDA ${ }^{\circledR}$, acrónimo de Tracking, Analysing, Imaging, Deciding y Acting (ver Figura 3).

Como ya se mencionó, para cada uno de los enfoques anteriormente enunciados, es posible utilizar una serie de técnicas y metodologías de apoyo para la consecución del objeto focal planteado en la fase inicial de la metodología. Para tal fin, de manera general (por mencionar algunos métodos y técnicas), los diferentes tipos de metodologías y técnicas pueden clasificarse de la siguiente manera (Heatman, 1969, Godet et al, 2006, Godet, 2000 y Lindgren, 2009):

1) Métodos basados en medios: Escaneo de medios, grupos de rastreo de tendencias, medios visuales, análisis de palabras clave, análisis de contenidos, etc.

2) Métodos basados en entrevistas: Método Delphi, conversaciones Delphic: entrevistas estructuradas, datos a largo plazo, grupos focales, paneles, grupos creativos (futuros), diálogos futuros, estudios futuros participativos, etc.

3) Métodos basados en líneas de tiempo: Desarrollo basado en formas o vías arquetípicas, extrapolación de tendencias a través de un análisis basado en una línea de tiempo y análisis multivariado, analogías, tendencias a largo plazo, curva s, el surgimiento de nuevos paradigmas, impactos cruzados probabilizados SMIC-prob-expert, etc. 
4) Métodos de generación intuitiva: Lluvia de ideas (brainstorming), construcción intuitiva de líneas de tiempo, encabezados y pósteres, imaginación, historias futuras, paradojas, etc.

5) Métodos orientados a actores: análisis de actores/análisis de competidores, observación de la competencia y análisis de la cadena de valor, análisis de impacto simple (SIM, Single-Impact Analysis), etc.

6) Métodos enfocados a consecuencias: E1 análisis morfológico, administración de tópicos, los árboles de competencia, los árboles de pertinencia, producción de eventos futuros, la probabilidad de los efectos, el árbol de trayectorias, etc.

7) Métodos sistémicos: Análisis de la complejidad y la incertidumbre, análisis de impactos cruzados, el análisis estructural, el método MACTOR, análisis de sistemas, mapeo causal/ diagramas de lazos o relaciones causales y el análisis de sistemas como una herramienta de aprendizaje, etc.

8) Métodos multicriterio: Promethee GAIA, ELECTRE, MACBETH, entre otros.

De acuerdo a lo anterior, la creación de escenarios a través de un método definido o no, es más que la aplicación mecánica de una serie de pasos ya establecidos. La planeación normativa o prospectiva es más que la suma o divergencia de diferentes tipos de enfoques (francés, nórdico, entre otros), más bien es un campo en el que confluyen todos los tipos de planeación conocidos: operativa, estratégica, normativa y contingente en donde éstas se entrelazan y se complementan unas a otras, de tal forma que conviven de manera simbiótica bajo la estructura de un objetivo focal, el cual es estructurado y definido de acuerdo a las necesidades de un sujeto o ente, que busca intervenir o cambiar su entorno inmediato, buscando con ello resolver sus necesidades o carencias de la mejor manera posible.

\section{Identificación de escenarios variables y clústeres}

Inicialmente dentro de la construcción de escenarios, generalmente surgen dos problemas importantes. El primero, al momento de la concepción del objeto focal; y el segundo, en la fase previa al desarrollo de los escenarios (identificación y correlación de variables). En relación al primer problema se puede establecer que en la actualidad no existe una categorización bien definida de los tipos de escenarios que pudiesen presentarse, lo que da a lugar a que se utilice una cantidad de tiempo importante en la definición del objeto focal del estudio en cuestión, para determinar el tipo de técnica o método requerido; aunque algunos autores han definido en promedio alrededor de dos o tres (Van der Heiden, 2006; Godet, 2006 y Ringland, 2002) categorías, dentro de las cuales se pueden enmarcar los escenarios en la actualidad.

Una correcta identificación del tipo de escenario a requerirse, permite y facilita, tanto la identificación del objeto focal del estudio, como el establecimiento de los alcances de éste. Sin embargo, en ocasiones el establecimiento del objeto focal del estudio (cuando no se tiene 
la claridad adecuada de lo que se pretende) podría resultar una tarea extenuante, sobre todo porque en esta etapa o fase no sólo se define el marco de referencia sobre el cual se desarrollará la planeación prospectiva, sino que también influye directa o indirectamente en los resultados a obtenerse. Es por ello que en una primera propuesta los escenarios prospectivos/ normativos podrían clasificarse de acuerdo a tres categorías básicas, así: (1) Por su forma de construcción; (2) Por el tipo de planeación que se pretende utilizar para alcanzar una posición competitiva; y finalmente, (3) Por su intención de desarrollo. En relación al primer tipo (por su forma de construcción) los escenarios pueden clasificarse en al menos cuatro tipos:

1) Escenarios proyectivos: Los escenarios proyectivos están basados en conclusiones generales, las cuales son trazadas a partir de la experiencia o de evidencia experimental o empírica. En este tipo de escenarios se parte de lo particular a lo general. Los escenarios proyectivos pueden estructurarse a nivel de eventos o a nivel de estructuras. La construcción de la forma proyectiva en ocasiones puede generar que los escenarios no posean una claridad adecuada (en una primera aproximación) que permita distinguir de forma clara un escenario de otro. Es por lo anterior que se deben interrelacionar adecuadamente todos aquellos elementos que entran en juego al momento de la construcción de los escenarios. Generalmente, este tipo de escenarios contempla una narrativa de los eventos esperados (eventos plausibles) interrelacionando cada una de las variables más relevantes que contribuyen de forma importante a incrementar o propiciar un ambiente de incertidumbre dentro del entorno en cuestión.
2) Escenarios deductivos: Este tipo de escenarios parte de lo general a lo particular. Normalmente se inicia de un estado final o evento tope, a partir del cual se van construyendo, deduciendo e identificando aquellos sucesos o acontecimientos que deben o deberían ocurrir, y que por los cuales se podría obtener el resultado deseado (factores clave y fuerzas motrices). Generalmente, este tipo de construcción de escenarios se combina con metodologías de identificación de actores y estrategias, con la finalidad de obtener un panorama completo de la situación y de los posibles cursos de acción a implementar.

3) Escenarios incrementales: No todas las entidades o agentes se encuentran preparados para este tipo de escenarios (proyectivos y deductivos), sobre todo si éste es el primer contacto con este tipo de metodologías (escenarios). Para ello se recomienda el desarrollo de algunas de las tendencias identificadas a partir el futuro oficial (proyecciones, estimaciones, plan de acción, proyectos, entre otros) para luego desarrollar escenarios o futuros alternos a partir de éste, tratando de identificar aquellos factores clave e incertidumbres que pongan en riesgo las estrategias de la organización (Van der Heijden, 2006).

4) Escenarios o estados idealizados: Los escenarios o estados idealizados resultan ser los más complejos en cuanto a su construcción. Los estados idealizados requieren de una capacidad de abstracción generalmente grande, esto es porque inicialmente se debe de concebir una idealización del estado al que se pretende llegar; para esto se requiere 
de alguna forma la construcción de escenarios proyectivos con la finalidad de dilucidar el posible curso de acción que pudieran tomar los eventos para alcanzar dicho escenario. Posteriormente, una vez concebido el estado idealizado, se debe retroceder en el tiempo, de forma deductiva, con el objeto de identificar las acciones necesarias y los actores que deberían implicarse para alcanzar dicho estado. Finalmente, se requiere suavizar el modelo para su implementación mediante aproximaciones sucesivas (de forma incremental), y el establecimiento de indicadores que permitan dar seguimiento a la ejecución de las estrategias necesarias para ello; así mismo, se requiere del desarrollo de un plan rector para su realización (se procede de igual manera cuando se opte por un cambio radical en la forma en que se realizan las cosas dentro de la organización, solamente que la forma de implementación, en este caso, no resulta ser tan sencilla (Akkoff, 2006).

De la misma forma, los escenarios normativos/ prospectivos pueden clasificarse de acuerdo al tipo de planeación que se pretende utilizar para alcanzar una posición competitiva (Ringland, 2002), así (ver Cuadro n. ํ):

1) Planeación enfocada: La construcción de escenarios mediante la planeación enfocada se fundamenta en el desarrollo de estrategias basadas solamente en un escenario, sobre el cual giran las acciones futuras de la organización o entidad.

2) Planeación robusta: La construcción de escenarios mediante la utilización de una planeación robusta se basa en la generación de estrategias a partir de múltiples escenarios, por lo cual la estrategia a desarrollar deberá ser viable dentro de todos los escenarios identificados.

Finalmente, los escenarios normativos se pueden categorizar de acuerdo a su intención de desarrollo, como pueden ser así:

1) Exploratorios: Se refieren a aquellos escenarios que tienen como punto de referencia el presente, tomando en cuenta las tendencias actuales y los posibles eventos presentes y futuros, para lo cual se desarrollan los posibles escenarios futuros, considerando sus posibilidades y su plausibilidad, con el fin de explorar el futuro posible a partir de unas condiciones partida dadas. Este tipo de escenarios se puede subdividir en dos categorías, como son:

i. Tendencial: Su objeto principal es el de identificar un futuro posible, asumiendo la permanencia y la predominancia de las tendencias más fuertes y examinado la continuidad de las tendencias en el futuro.

ii. Enmarcado o de contraste: Su objeto principal es el de delimitar el espacio de los futuros plausibles. Este tipo de escenario asume la permanencia y la predominancia de ciertas tendencias, variando en forma extrema las hipótesis concernientes a la evaluación de éstas.

2) Anticipatorios: Se refieren a la construcción de escenarios, tomando en cuenta el comportamiento y el desempeño pasado y presente, con el objeto de generar diversos futuros plausibles, con la intención de 


\section{Cuadro n. ${ }^{\circ} 1$}

Clasificación de los escenarios de acuerdo al tipo de planeación utilizada.

\begin{tabular}{|c|c|c|c|c|c|c|}
\hline & & Variante 1 & Variante 2 & Variante 3 & Variante 4 & Variante 5 \\
\hline 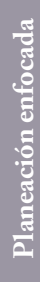 & 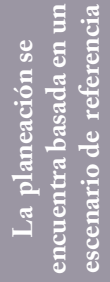 & $\begin{array}{c}\text { La estrategia } \\
\text { se encuentra } \\
\text { basada en } \\
\text { el escenario } \\
\text { con mayor } \\
\text { probabilidad } \\
\text { de ocurrencia. }\end{array}$ & $\begin{array}{l}\text { La estrategia } \\
\text { está basada en } \\
\text { el escenario } \\
\text { más riesgoso. }\end{array}$ & $\begin{array}{l}\text { Estrategias } \\
\text { reactivas y/o } \\
\text { preventivas. }\end{array}$ & $\begin{array}{l}\text { La estrategia } \\
\text { está basada en } \\
\text { el escenario } \\
\text { con mayores } \\
\text { oportunidades. }\end{array}$ & $\begin{array}{l}\text { La estrategia } \\
\text { está basada en } \\
\text { el escenario } \\
\text { deseado. }\end{array}$ \\
\hline 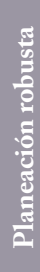 & 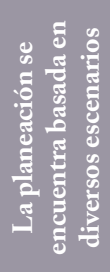 & $\begin{array}{c}\text { La seguridad } \\
\text { de la estrategia } \\
\text { se encuentra } \\
\text { basada sobre } \\
\text { el escenario } \\
\text { con mayor } \\
\text { probabilidad } \\
\text { de ocurrencia. }\end{array}$ & $\begin{array}{l}\text { La estrategia se } \\
\text { concentra en la } \\
\text { minimización } \\
\text { de riesgos. }\end{array}$ & $\begin{array}{c}\text { La estrategia se } \\
\text { concentra en la } \\
\text { maximización } \\
\text { de la } \\
\text { flexibilidad. }\end{array}$ & $\begin{array}{l}\text { La estrategia se } \\
\text { concentra en la } \\
\text { maximización } \\
\text { de } \\
\text { oportunidades. }\end{array}$ & $\begin{array}{c}\text { La seguridad } \\
\text { de la estrategia } \\
\text { se basa en } \\
\text { el escenario } \\
\text { deseado. }\end{array}$ \\
\hline
\end{tabular}

Fuente: Adaptado de Ringland, 2002.

prepararse de forma proactiva y preactiva ante la posible ocurrencia de dichos futuros, mediante el desarrollo de estrategias que den respuesta a las necesidades y que potencien las áreas de oportunidad (debilidades) dentro de la organización/entidad. Dentro de los escenarios anticipatorios se tienen los siguientes:

i. Idealizados: Este tipo de escenarios representa imágenes de un futuro deseable. El primer paso consiste en construir una imagen deseada del futuro (imagen objetivo). El segundo paso consiste en desarrollar el marco de eventos que tendrían que acontecer para alcanzar la situación deseada y, finalmente, se desarrollan las estrategias requeridas para alcanzar la imagen objetivo. Desde esta perspectiva el escenario futuro estará en función no sólo del presente y del pasado, sino que también de lo que se desea, incorporando al mismo tiempo las acciones y los eventos requeridos para el alcance de dicho estadio.

ii. Prospectivos: Los escenarios prospectivos se construyen a partir de situaciones pasadas y presentes (comportamiento y desempeño), con el objeto de construir las situaciones futuras mediante la interrelación de variables y tendencias mediante el uso de las capacidades imaginativas de los interesados. Lo que se busca es la identificación de la situación futura que presente el mayor grado de plausibilidad o una mezcla de éstas, con la finalidad de prepararse de la mejor manera posible ante un futuro incierto, pero plausible, mediante el desarrollo de las estrategias necesarias para tal fin. 
Una identificación acertada del tipo de escenario requerido implica definir de forma previa el objeto focal del estudio, aunque no de manera formal; pero sí, al menos, grosso modo, con la intención de desarrollo del ejercicio, dando lugar a la facilitación y a la ejecución del proceso de planeación a través de escenarios, por medio de la identificación temprana, tanto del objeto y como del alcance de las actividades a realizarse.

El segundo de los problemas dentro de la construcción de escenarios se presenta en la fase previa al desarrollo de los escenarios, específicamente en la etapa de identificación y correlación de variables. Invariablemente del tipo de método o técnica que se utilice para la construcción de los escenarios se deben identificar las variables/fuerzas y/o tendencias que direccionen el sentido de uno y/o más escenarios, así como los actores que intervienen o intervendrán en el futuro posible. Sin embargo, en ocasiones la identificación de las variables o fuerzas resulta ser una actividad no tan trivial, ya que éstas generalmente dependen de otros factores o subvariables, las cuales si son ignoradas se convierten en elementos discordantes dentro del estudio, ya que la plausibilidad de un escenario en particular, se ve disminuida por la carencia de dichos elementos generadores de tendencias o puntos de inflexión que a la postre resultan ser determinantes.

En este contexto la correcta identificación del grado de dependencia o interrelación entre variables/fuerzas depende de la información disponible, del contexto y, sobre todo, de la capacidad analítica del proyectista. La información disponible, en ocasiones, no es la suficiente o se encuentra incompleta, por lo que el proyectista deberá de poseer la habilidad de identificar la importancia relativa entre variables/ factores y las interrelaciones que pudieran subyacer en el contexto de la situación actual y/o pretérita. Es en este punto donde la situación se torna crítica, ya que si no se posee la claridad necesaria en la forma en que un escenario puede modificarse de acuerdo al sentido o variación de una variable, entonces el el proyectista carece de una visión holística y sistémica de la situación o del contexto en cuestión.

Por las razones ya expuestas, es posible afirmar que para proyectar un futuro plausible, se hace necesario comprender el pasado y el presente, identificando la interrelación que guardan las variables/fuerzas y los actores entre sí, y cómo éstos dan origen a las tendencias (Lindgren, 2009) observables en el presente inmediato $\mathrm{y}$ en futuro posible. Para ello se requiere del agrupamientos de variables afines a través de criterios, propiedades o características que permitan encontrar puntos de coincidencia para la obtención de un mapeo relacional tendiente a la conformación de la base sobre la que se partirá, para el desarrollo de las proyecciones futuras, siendo esto posible de alcanzarse a través de la definición y agrupamiento variables/fuerzas por medio de un análisis de clústeres.

Clúster es un término que puede definirse así: (1) Una concentración, conjunto, o aglutinamiento pequeño de elementos, ítems, etc., en torno a un punto central (Verbeek, 1999); y como (2) Número de cosas, ítems, etc., del mismo tipo que crecen/desarrollan/expanden de forma estrecha y conjunta (Verbeek, 1999). Aunque las bases de su origen se remontan con Marshall en 1890 (Sedita et al, 2012), la noción de clúster más aceptada y a 
la vez precursora de su expansión hacia otras áreas como son la política, la economía, la tecnología, la competitividad, etc. (Sedita et al, 2012), es la introducida por Michael Porter (1990 y 2001), en la que establece que un clúster es un agrupamiento como tal (refiriéndose a los clústeres industriales) en donde éste es una concentración de entidades relacionadas entre sí, que se encuentran próximas geográficamente, las cuales colaboran de forma estrecha para alcanzar grados mayores de competitividad (Hefner, 2009 y Soriano, 2008). Siendo posible encontrar dos tipos de enfoques: (1) El enfoque de los clúster basados en su similitud; y (2) El enfoque de clústeres basados en su interdependencia (Verbeek, 1999).

En este sentido, aunque la modelación dinámica de sistemas es utilizada para la representación y simulación de diversos modelos (Lee et al, 2012; Kasperek et al, 2014; Liu et al, 2015, Lee eta al (2012) y Neuwirth et al, 2015), análogamente, el análisis de clústeres es posible realizarse a través de la modelación de la dinámica de sistemas, ya que de alguna forma ya existen ciertos arquetipos identificados dentro del análisis de sistemas (Sengue, 1990; Anderson et al, 1997; Lannon, 2008 y Soriano, 2008). Sin embargo, más que arquetipos, se hace necesario analizar la estructura de estos modelos (Lehman, 2011), con el propósito de determinar si las estructuras mismas (arquetípicas o no) son capaces de generar una superficie respuesta plausible o adecuada a la situación o contexto, siendo esto posible si se dan las condiciones siguientes:

a) Todas las fuerzas/variables y subfuerzas/ subvariables locales y no locales son susceptibles de ser representadas a través de lazos causales. b) Las fuerzas/variables son susceptibles de ser agrupadas en clústeres para su análisis, en donde el peso de las subvariables determina el sentido/ dirección de la tendencia/respuesta/ salida de un escenario generado a través de lazos causales.

c) El entramado de la red causal permite representar una situación en particular permitiendo no solamente la depuración de variables/fuerzas dentro de la construcción base, sino que también observar la coherencia e interrelación de la construcción como un paso previo al desarrollo de los escenarios.

El que todas las fuerzas/variables y subfuerzas/ subvariables locales y no locales pueden ser representadas a través de lazos causales, conlleva a que todos los sistemas, ya sean estos naturales o creados por el hombre, son susceptibles de ser representados a través de la dinámica de sistemas. Entonces, partiendo del principio de la teleología de los sistemas, ${ }^{1}$ el arreglo del sistema obedece a dicho propósito; el sistema, entonces, no puede ser concebido como un conjunto de elementos dispuestos de forma aleatoria, ya que de otra manera el modelo podría no ser útil en la representación de la situación actual, ni tampoco para una proyección futura. Así mismo, no se puede dividir un sistema en partes disociadas bajo un supuesto reduccionista; el sistema debe guardar su integralidad y debe ser visto de forma holística. De igual forma, tampoco pueden asociarse sistemas sin relación alguna y pretender obtener un sistema coherente

$1 \mathrm{E}$ l sistema en cuestión debe de analizarse y concebirse desde una perspectiva teleológica. 
de los sistemas previamente identificados. De esta manera se puede afirmar que todos los sistemas a considerarse en el análisis deben ser homeostáticos. Cabe destacar que la representación del mapa del entramado causal es un proceso iterativo que debe ir perfeccionándose cada vez más a través de la reflexión constante sobre el modelo y las variables que intervienen en el proceso.

Las relaciones que se generan dentro de un lazo causal deben ir adecuadamente identificadas, como son el balance, el reforzamiento, etc. (ver Figura n. ${ }^{\circ}$ ), ya que en la actualidad, si bien es posible modelar un sistema sin identificar previamente los clústeres (Beng, 2012; Feng, 2012 y Chao et al, 2013), la delimitación permite no sólo establecer las fronteras, sino que también determinar los conectores, puentes e interrelaciones entre variables que, de una u otra forma permiten comprender e interiorizar la situación de una mejor manera para determinar los resultados o las trayectorias de dependencia en la construcción de los escenarios.

Desde este esquema, entonces, es posible agrupar las fuerzas/variables en clústeres para su análisis tanto de pertinencia como de interrelación, tomando en cuenta que, de acuerdo al dinamismo del entorno, éstas cambiarán su valor a través del tiempo. Es por ello que para la construcción de escenarios se requiere de la correcta identificación de variables/fuerzas que intervienen en el contexto de interés. En una primera fase se debe realizar un barrido de todas las variables/fuerzas y tendencias que van emergiendo a medida que se empieza a documentar la situación actual y pasada, a través de un inventario - sin un orden en particular- con la finalidad de barrer todo el espectro de posibles eventos, variables, causas, fuerzas, efectos y tenencias sin identificar categoría alguna. Una vez elaborado el inventario se empieza a identificar cada una de las categorías anteriores. ${ }^{2}$ A partir del inventario se agrupan las variables/fuerzas/eventos por su afinidad o porque éstas resultan ser subvariables/ sub-fuerzas que dan forma, incrementan o disminuyen a una variable/evento/fuerza en particular.

Si bien es cierto que una variable/fuerza puede tener diversos componentes, sub-variables/ subfuerzas, no todos los componentes poseen el mismo peso o la misma importancia; el peso o importancia resulta determinante al momento de dar forma o trayectoria de salida, ya que dependiendo del peso del evento/variable/fuerza, será la dirección que tome éste (ver Figura n. ${ }^{\circ}$ ). Cabe señalar que en realidad se deben efectuar dos categorizaciones: (1) Hacia el interior del clúster propiamente dicho; y (2) Hacia el exterior de los clústeres.

La primera de las categorizaciones tiene como fin la de identificar las subvariables/subfuerzas que poseen la mayor ponderación/peso, las cuales determinarán el curso/trayectoria del clúster. La segunda categorización se encuentra relacionada con el peso de un clúster específico en relación a los demás; en donde la trayectoria y/o fluctuación de la tendencia se define por el sentido y el peso del conjunto de todos los clústeres, es decir, que a partir de la influencia (positiva o negativa) en la trayectoria de

\footnotetext{
2 Aunque en esta primera fase solamente se requiere agrupar los eventos, fuerzas o variables, es recomendable identificar también las causas y las tendencias ya, que posteriormente se utilizarán para el desarrollo de los escenarios.
} 


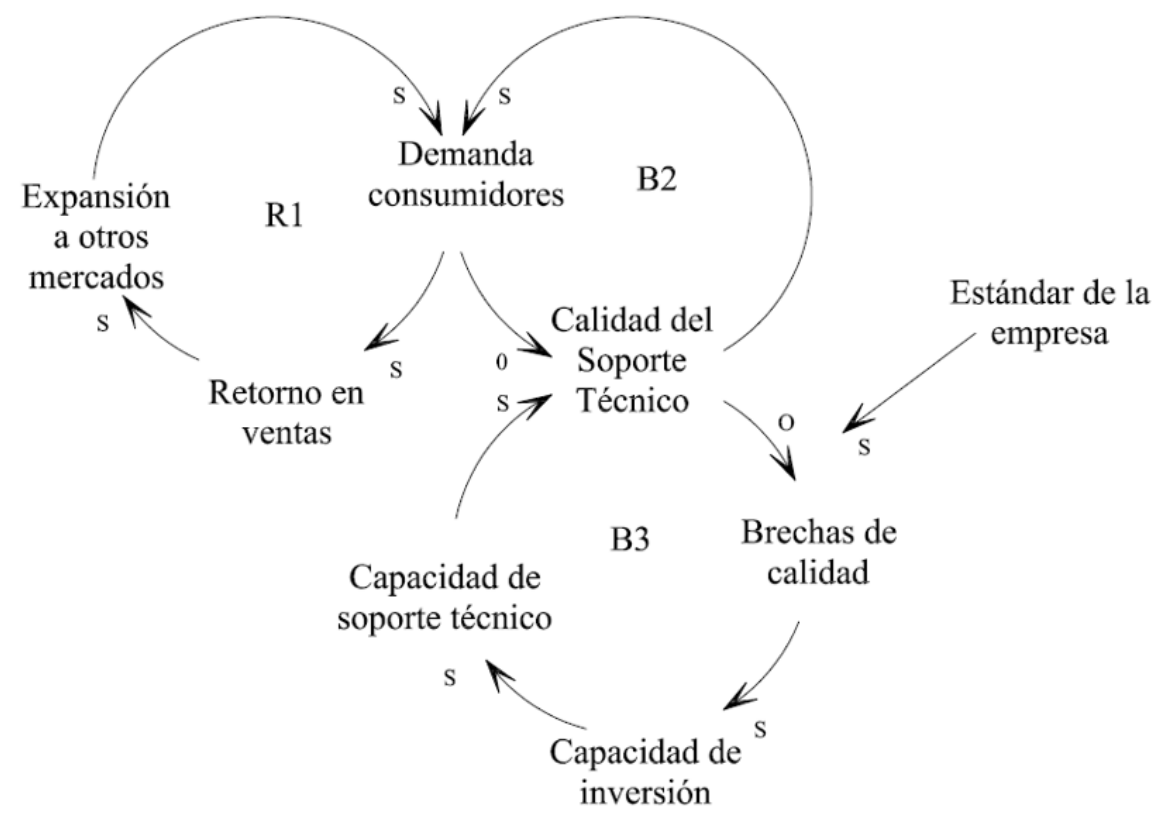

Figura n. 4. Lazo causal: Desinversión en el servicio. Fuente: Adaptado parcialmente de Anderson et al, 1997

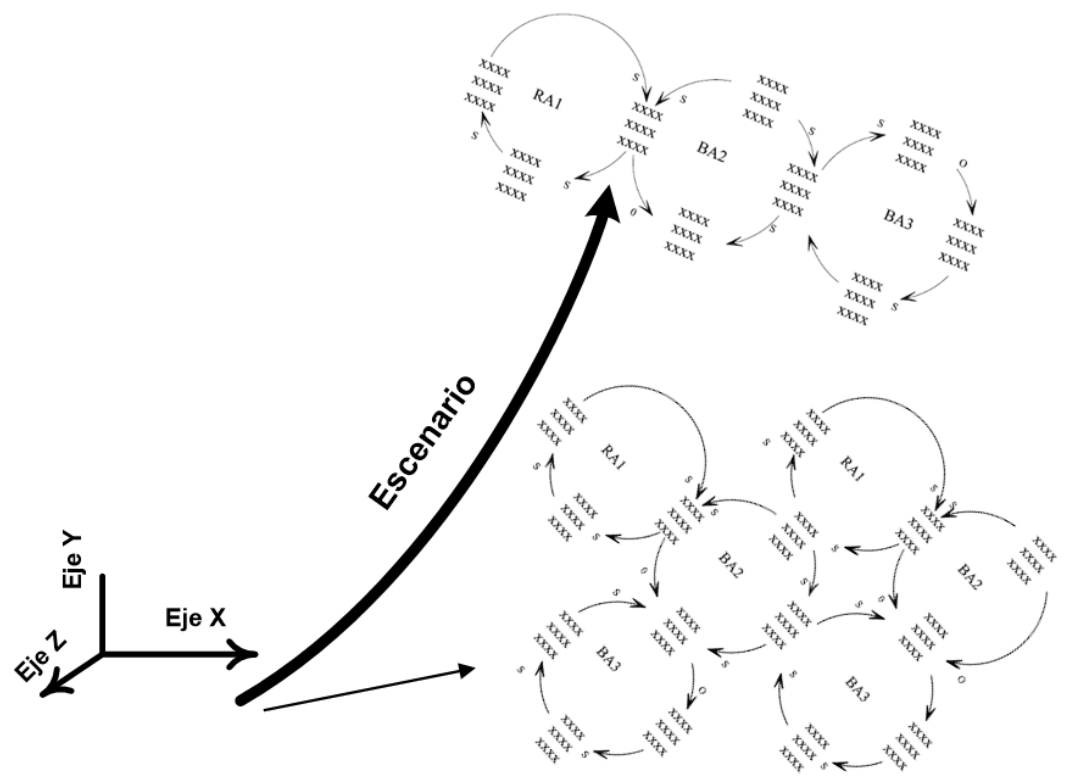

Figura . $^{\circ}$. Clúster de variables. Fuente: Elaboración propia. 
la tendencia, los clústeres determinarán el sentido y la lógica del escenario, en donde el peso específico de cada clúster contribuye a la conformación de un escenario dado.

$\mathrm{Si}$ se supone que los clústeres pueden representarse en un plano bidimensional (espacio donde interactúan las variables/fuerzas), los escenarios entonces serían una proyección en un plano ortogonal al plano de las variables, por lo que se tendría una representación tridimensional, en la cual se observarían las fluctuaciones, cambios de dirección y sentido de la tendencia provenientes de la influencia de las variables/fuerzas agrupadas. Al mismo tiempo, puede verificarse que si se obtuviera un corte trasversal de la tendencia de un escenario en alguna sección de la proyección (ver Figura n. ${ }^{\circ}$ ) sería posible encontrar un entramado base en ese instante de tiempo t, distinto o similar al inicial, pero no igual al entramado base que originó la tendencia/escenario. Entonces, por ejemplo, si se considera desarrollar un escenario idealizado, la imagen objetivo (el estado final) sería el punto de partida para elaborar el entramado inicial, debido a que se partiría de algo a lo que se aspira, se tendría que desarrollar en retrospectiva todas aquellas acciones/fuerzas/variables que tendrían que estar presentes para poder conseguir el resultado deseado.

Las fuerzas/variables locales, como las externas, poseen cierto grado de interrelación, el cual a su vez viene dado a través de los conectores de enlace. Si un escenario no es coherente, se verá reflejado en el diagrama de construcción base, ya que no existirán conexiones entre variables/ fuerzas. Por el contrario, a mayor existencia de conectores existirá una correlación expresa entre las variables/fuerzas y los escenarios. A estos conectores se les denominará conectores de enlace y su función justamente es correlacionar las diversas variables/fuerzas que intervienen en un escenario, en donde, cuanto mayor sea el número de conectores de enlace, mayor será el grado de correlación e interrelación entre las variables de entrada y de salida (ver Figura n. ${ }^{\circ}$ ). Estos conectores de enlace pueden presentar sentidos iguales o dispares, pero su verdadera importancia radica en que sirven de puente para la interacción del sistema a través de todo el entramado base. Es en este punto en donde la correlación de variables/fuerzas surge como elemento de gran importancia para dar origen a la correlación del escenario mismo.

El entramado de la red causal permite representar una situación en particular, permitiendo no sólo la depuración de variables/ fuerzas dentro de la construcción base, sino que también la observación de la coherencia de la construcción como un paso previo al desarrollo de los escenarios. Cuando los lazos causales de las diferentes variables/fuerzas se interrelacionan, es posible observar la coherencia del escenario o los escenarios y la pertinencia de las variables dentro de la proyección. La coherencia del entramado base resulta crucial para el desarrollo de escenarios congruentes y plausibles, es decir, la proyección de un escenario debe presentar un entramado con un razonamiento válido y verosímil, una vez que se ha partido de una razonamiento base a partir de variables/fuerzas que intervienen en una situación inicial, cimentada en el desempeño, actuación o hechos pasados /o presentes para la construcción de futuros alternativos dentro del campo de lo concebible. 
Ahora bien, lo concebible depende de la información con la que cuente el proyectista, además de la capacidad imaginativa de éste. Si no se cuenta con la debida o una mínima información para la construcción de escenarios, la proyección en sí carece de valor estratégico, ya que no podría abarcarse todo el espectro de posibilidades, y sobre éste decidir la plausibilidad de éstas. Sin embargo, la plausibilidad viene dada a partir de la construcción base y de la forma en que las variables/fuerzas se interrelacionan para crear una nueva realidad alterna (en la actualidad existen diversas técnicas y métodos, tanto cuantitativos como cualitativos para determinar la influencia y la importancia tanto de actores como de fuerzas/variables).

Es así como la coherencia del entramado base implica el concebir los escenarios como una entidad unitaria, donde los hechos secundarios aportan la información necesaria para alcanzar la lógica requerida a fin de determinar la plausibilidad o no de la situación futura y adquirir así la significancia adecuada para su consideración en el proceso de la toma de decisiones. La coherencia, entonces, se encuentra íntimamente ligada a la cohesión, ya que la cohesión es una propiedad que organiza las ideas/fuerzas/ variables en una secuencia lógica a partir de la causalidad. Es así como podría advertirse que un escenario prospectivo es un conjunto de clústeres relacionados entre sí, dispuestos en una secuencia determinada, que ofrecen un resultado o respuesta particular, de acuerdo al orden, disposición, interrelación, dirección, sentido, peso e importancia de las variables/ fuerzas que intervienen en un contexto o situación particular dada.
Por lo tanto, la determinación del objeto focal de un escenario dado se encuentra íntimamente relacionada con la identificación del tipo de escenario a proyectarse. $Y$ es de esa forma donde, de acuerdo al tipo de escenario a proyectarse, serán los métodos y técnicas a utilizarse para construir el entramado base. De igual manera, es posible afirmar que la interrelación del entramado base, depende de la coherencia y de la verosimilitud de los eventos futuros, derivados del presente y del pasado, de las interconexiones e interrelaciones entre clústeres, así como de la disposición de los componentes, fuerzas/ variables y subfuerzas/subvariables hacia el interior del conjunto de clústeres.

Entonces es así que la construcción de escenarios prospectivos/normativos debe conllevar a la revisión de una serie de elementos necesarios para el aseguramiento y la verificación de la plausibilidad de éstos, como son los siguientes: (1) La estructura y la disposición de los clústeres dentro del sistema, las relaciones (internas y externas) entre clústeres; (2) El propósito del sistema y del estudio, (3) La tendencia del sistema al equilibrio, la integración del sistema; (4) La información previa y la actual del sistema; (5) El peso de las variables/fuerzas dentro del sistema y, sobre todo, (6) El sentido de los lazos causales y la cantidad de conectores de enlace entre clústeres; sin menoscabo de otro tipo de factores que pudieran agregarse para la obtención de proyecciones futuras de un evento dado, a partir de unas condiciones presentes y futuras, las cuales determinaran los eventos y los resultados a obtenerse en un horizonte de tiempo determinado. 


\section{Observaciones generales}

La selección del tipo de escenario a utilizarse conlleva a seleccionar de una mejor forma el tipo de métodos, técnicas y recursos que se deben utilizar para la construcción de futuros alternativos, ya que permite identificar de forma temprana el objeto focal, los alcances y el objetivo de forma más expedita, a través de una clasificación definida de acuerdo a las necesidades del proyectista.

La agrupación y la delimitación de variables/ fuerzas debido a su afinidad, correspondencia y/o subordinación, con fronteras bien definidas y demarcadas en clústeres, permiten el apropiamiento de una situación base, ya que al momento de construir y definir los límites, en realidad se define el contexto, las interconexiones, las interrelaciones y los actores que intervienen en el desarrollo de situaciones o proyecciones futuras, a partir de un contexto dado. Es así como mientras mayor sea el conocimiento de la estructura sobre la cual se monta un escenario dado (y de sus actores también), es posible obtener proyecciones del futuro que sean plausibles $y$, consecuentemente, diversos tipos de estrategias encaminadas a administrar la incertidumbre de los posibles eventos futuros.

La estructura de un entramado base, por sí mismo, no es un escenario; un escenario cobra su significado y su significancia solamente cuando entran en juego las interrelaciones entre los actores del entorno como tal, es decir, esto ocurre justamente en el instante en que se yuxtaponen todas las variables (y donde la estructura y los sujetos se interrelacionan), entrelazándose las fuerzas/variables, condicionantes, tendencias y actores para interactuar de una forma dinámica en la construcción de un futuro alternativo, pero plausible; no pudiéndose entender dicho futuro sin su componente presente y pasado, que den forma y justifiquen los posibles resultados futuros de manera correcta o al menos creíble.

La creación de escenarios a través de un método definido o no, es más que la aplicación mecánica de una serie de pasos ya establecidos. La creación de escenarios implica mantener una actitud crítica y reflexiva ante la realidad, con el objeto de desentramar o tejer (según sea el caso) las causas y consecuencias últimas de un futuro posible o alternativo, pero plausible; dicha actitud debe conllevar, además, a no dar nada por hecho y a tratar de identificar de manera efectiva las fuerzas o variables que influencian o propician de una manera u otra, la posibilidad de ocurrencia de una nueva realidad alterna.

El éxito o el fracaso de una metodología o técnica (o una combinación de éstas) depende en gran medida de la cantidad de información que se posea para el desarrollo del análisis situacional y la definición y establecimiento del objeto focal del estudio; ya que sin esta información no se es capaz de definir en primera instancia la estructura y la complejidad del objeto ni los esfuerzos que deben comprometerse por parte del sujeto para llevar a cabo el proceso de planeación prospectiva de la mejor manera posible.

\section{Bibliografía}

Anderson V. y Johnson L. (1997). Systems Thinking Basics: From Concepts to Causal Loops. Pegasus Communications Inc. USA. 
Ackoff R. (2006). Planificación de la empresa del futuro, Editorial Limusa, Noriega Editores, México DF.

Beng D. (2012). Modeling the Impact of Anchoring in Capacity Adjustments to WorkIn-Process Behavior in a Two Stage Production System: A System Dynamics Approach Procedia - Social and Behavioral Sciences 57 pp 566-574.

Chao Y, and Zishana M. (2013). System Dynamics Model of Shanghai Passenger Transportation Structure Evolution Procedia - Social and Behavioral Sciences 96, pp 11101118.

Fahey L. y Randall R. (1998). Learning From the Future: Competitive Foresight Scenarios, Published by John Wiley \& Sons Ltd., US.

Feng Y. (2012). System Dynamics Modeling for Supply Chain Information Sharing, Physics Procedia 25, pp. 1463-1469.

Godet M. (2000). The Art of Scenarios and Strategic Planning: Tools and Pitfalls, Technological Forecasting and Social Change 65, pp. 3-22.

Godet M., Monti R., Meunier F. and Roubelat F. (2000). La Caja de Herramientas de la Prospectiva Estratégica, Gerpa, 4. ${ }^{\text {a Edición. }}$

Godet M. (2006). Creating Futures. Scenario Planning as a Strategic Management Tool, Economica Ltd., France.

Heatman F. (1969). THE LANGUAGE OF FORECASTING, futuribles, Paris, France.
Hefner F.(2009). Cluster theory: a new prescription for old style government planning?, in: Unleasing capitalism: a prescription for economic prosperity in South Carolina. South Carolina: South Carolina Policy Council education.

Kasperek D., Chucholowski N., Maisenbacher S., Lindemann U. and Maurer M. (2014). A Method for Impact Analysis of Cyclic Changes within Innovation Processes of PSS. Product Services Systems and Value Creation. Procedia CIRP 16, pp $205-210$.

Lannon C. (2008). A BEGINERS'S GUIDE TO SYSTEMS THINKING. Pegasus Communications Inc. USA.

Lehman S. (2011). What can be learned from cluster evaluation studies for cluster theory and future cluster policy? 56th Annual ICSB (International Council for Small Bussiness) Word Conference.

Lee Ch., and Chungb Ch. (2012). An Inventory Model for Deteriorating Items in a Supply Chain with System Dynamics Analysis, Procedia - Social and Behavioral Sciences 40, pp 41 - 51 .

Lindgren M. and H. Bandhold. (2009). Scenario Planning: The Link between Future and Strategy, Edited by PALGRAVE MCMILLAN, New York.

Liu C., Xie Z., Sun F. y Chen L. (2015). System dynamics analysis on characteristics of iron-flow in sintering process. Applied Thermal Engineering Volume 82 pp. 206-211. 
Miklos T. and M. Tello. (2007). Planeación prospectiva: Una estrategia para el diseño del futuro, Centro de Estudios Prospectivos de la Fundación Javier Barrios Sierra A.C., Editorial LIMUSA, Grupo Noriega Editores, México.

Neuwirth C., Peck A. and Simonovi S. (2015). Modeling structural change in spatial system dynamics: Daisyworld example. Environmental Modelling \& Software 65 pp. 30 - 40.

Ogburn, W. (1937). Technological Trends and National Policy, Including the Social Implications of New Inventions, National Policy and Technology, in US National Resources Committee, Washington: USGPO: pp. 3-14.

Polak F., (1973). The Image of the Future, Elsevier Scientific Publishing Company, Amsterdam, Netherlands.

Porter M. (1990). The Competitive Advantage of Nations. Ney York: The Free Press.

Porter, M. (2001). Clusters of Innovation: Regional Foundations of U.S. Competitiveness. Washington, DC: The Council on Competitiveness.

Ringland G. (2002). Scenarios in Business, John Wiley and Sons, Baffins Lane, Chichester, UK.

Sedita S.R. (2012). The Birth And The Rise Of The Cluster Concept, Druid, June, Copnehagen, Denmark.
Sengue P. (1990). The Fifth Discipline: The Art and Practice of The learning Organization. Currency Dobleday Publishing Group Inc., New York.

Swhartz P. (1996). The Art of the Long View: Planning in an Uncertain World, Edited by DOUBLEDAY, New York.

Soriano M. (2008). El papel del Emprendedurismo en la formación de Clusters Industriales. Trabajo de tesis. Universidad de las Américas, Escuela de Economía y Negocios, Puebla, México.

The Centre of Strategic Business Studies, CSBS. (1999). Putting scenarios, at the heart of strategy, The Antidote, Scenario: The search for foresight, Issue: 22, pp. 29-31, Hampshire, UK.

Van der Heijden K. (2009). The art of Strategic Conversation, Edited by John Wiley \& Sons Ltd., England.

Verbeek, H. (1999). Innovative Cluster. Tesis doctoral, Erasmus Unniversiteit te Rotterdam, disponible en: www.oecd.org/sti/inno/2098804. pdf

Vergara J., T. Fontalvo y F. Maza. (2010). La planeación por escenarios: Revisión de conceptos y propuestas metodológicas, Prospectiva, Vol. 10, No. 2, Julio-Diciembre, pp. 37-42. 